\title{
A single exponential BKM type estimate for the 3D incompressible ideal MHD equations
}

Jianli Liu', Fenglun Wei ${ }^{1}$ and Kejia Pan ${ }^{2,3 *}$

${ }^{\text {*Correspondence: }}$

pankejia@hotmail.com

${ }^{2}$ School of Mathematics and Statistics, Central South University,

Changsha, 410083, P.R. China

${ }^{3}$ Key Laboratory of Metallogenic

Prediction of Nonferrous Metals,

Ministry of Education, Central South

University, Changsha, 410083,

P.R. China

Full list of author information is

available at the end of the article

\section{Abstract}

In this paper, we give a Beale-Kato-Majda type criterion of strong solutions to the incompressible ideal MHD equations. Instead of double exponential estimates, we get a single exponential bound on $\|(u, h)\|_{H^{5}}\left(s>\frac{5}{2}\right)$. It can be applied to a system of an ideal viscoelastic flow.

MSC: 35B65; 76W05

Keywords: MHD equations; ideal viscoelastic flow; Beale-Kato-Majda criterion; single exponential bound

\section{Introduction}

In this paper, we will get the Beale-Kato-Majda type criterion for the breakdown of smooth solutions to the incompressible ideal MHD equations in $\mathbb{R}^{3}$ as follows:

$$
\left\{\begin{array}{l}
u_{t}+u \cdot \nabla u+\nabla\left(p+\frac{1}{2}|h|^{2}\right)=h \cdot \nabla h, \\
h_{t}+u \cdot \nabla h=h \cdot \nabla u, \\
\nabla \cdot u=0, \quad \nabla \cdot h=0, \\
t=0: \quad u=u_{0}, \quad h=h_{0},
\end{array}\right.
$$

where $x \in \mathbb{R}^{3}, t \geq 0, u$ is the flow velocity, $h$ is the magnetic field, $p$ is the pressure, while $u_{0}$ and $h_{0}$ are, respectively, the given initial velocity and initial magnetic field satisfying $\nabla \cdot u_{0}=0, \nabla \cdot h_{0}=0$.

Using the standard energy method [1], it is well known that for $\left(u_{0}, h_{0}\right) \in H^{s}\left(\mathbb{R}^{3}\right), s \geq 3$, there exists a $T>0$ such that the Cauchy problem (1) has a unique smooth solution $(u, h)$ on $[0, T]$ satisfying

$$
(u(t, x), h(t, x)) \in C\left([0, T] ; H^{s}\right) \cap C^{1}\left([0, T] ; H^{s-1}\right) .
$$

Recently, Caflisch et al. [2] extended the well-known result of Beale et al. [3] to the 3D ideal MHD equations. More precisely, they showed that if the smooth solution $(u, h)$ satisfies the following condition:

$$
\int_{0}^{T}\|\nabla \times u\|_{L^{\infty}} d t<\infty \quad \text { and } \quad \int_{0}^{T}\|\nabla \times h\|_{L^{\infty}} d t<\infty
$$

then the solution $(u, h)$ can be extended beyond $t=T$, namely, for some $T^{\star}>T,(u, h) \in$ $C\left(\left[0, T^{\star}\right) ; H^{s}\left(\mathbb{R}^{3}\right)\right) \cap C^{1}\left(\left[0, T^{\star}\right) ; H^{s-1}\left(\mathbb{R}^{3}\right)\right)$. Many authors also considered the blow-up cri-

\section{Springer}

○2014 Liu et al.; licensee Springer. This is an Open Access article distributed under the terms of the Creative Commons Attribution License (http://creativecommons.org/licenses/by/2.0), which permits unrestricted use, distribution, and reproduction in any medium, provided the original work is properly cited. 
terion of the ideal MHD equations in other spaces; see [4-6] and references therein. More recently, for the following incompressible Euler equations:

$$
\left\{\begin{array}{l}
u_{t}+u \cdot \nabla u+\nabla p=0 \\
\nabla \cdot u=0 \\
t=0: \quad u=u_{0}
\end{array}\right.
$$

with $\nabla \cdot u_{0}=0$, Chen and Pavlovic [7] showed that if the solution $u$ to (4) satisfies

$$
\int_{0}^{T}\left(\iota_{\gamma}(\tau)\right)^{-\frac{5}{2}} d \tau<\infty
$$

where $\iota_{\gamma}(t)=\min \left\{L,\left(\frac{\|\omega(t)\|_{C^{\gamma}}}{\left\|u_{0}\right\|_{L^{2}}}\right)^{-\frac{2}{2 \gamma+5}}\right\}, \omega=\nabla \times u$ and $\|\omega\|_{C^{\gamma}}=\sup _{|x-y|<L} \frac{|\omega(x)-\omega(y)|}{|x-y|^{\gamma}}$, then the solution $u$ can be extended beyond $t=T$. The quantity $\iota_{\gamma}(t)$ was introduced by Constantin in [8] (see also the work of Constantin et al. [9]). For the blow-up criterion of incompressible Euler equations, we refer to $[7,10]$ and references therein.

\section{Main results}

In this short note, we develop these ideas further and establish an analogous blow-up criterion for solutions of the 3D ideal MHD equations (1). More precisely, we can get the following theorem.

Theorem 2.1 Let $(u, h)$ be a solution to (1) in the class (2) for $s=\frac{5}{2}+\gamma$. Assume that

$$
\int_{0}^{T}\left(l_{\gamma}(\tau)\right)^{-\frac{5}{2}} d \tau<\infty
$$

where $l_{\gamma}(t)=\min \left\{L,\left(\frac{\|\omega(t)\|_{C \gamma} \gamma}{\left\|u_{0}\right\|_{L^{2}}}\right)^{-\frac{2}{2 \gamma+5}},\left(\frac{\|\Omega(t)\|_{C \gamma}}{\left\|h_{0}\right\|_{L^{2}}}\right)^{-\frac{2}{2 \gamma+5}}\right\}, \omega=\nabla \times u, \Omega=\nabla \times$ h and the definition of $C^{\gamma}$ as above. Then there exists a finite positive constant $C_{\gamma}=O\left(\gamma^{-1}\right)$ independent of $(u, h)$ and $t$ such that

$$
\|(u, h)\|_{H^{s}} \leq\left\|\left(u_{0}, h_{0}\right)\right\|_{H^{s}} \exp \left\{C_{\gamma}\left\|\left(u_{0}, h_{0}\right)\right\|_{L^{2}} \int_{0}^{t}\left(l_{\gamma}(\tau)\right)^{-\frac{5}{2}} d \tau\right\}
$$

holds for $0 \leq t \leq T$.

Remark 2.1 Using a similar method, we also can get the blow-up criterion result about ideal viscoelastic flow

$$
\left\{\begin{array}{l}
\partial_{t} u+u \cdot \nabla u+\nabla p=\sum_{k=1}^{3}\left(F_{k} \cdot \nabla\right) F_{k}, \\
\partial_{t} F_{k}+u \cdot \nabla F_{k}=\left(F_{k} \cdot \nabla\right) u, \\
\nabla \cdot u=0, \quad \nabla \cdot F_{k}=0, \\
t=0: \quad u=u_{0}(x), \quad F_{k}=F_{k 0}(x)
\end{array}\right.
$$

with $\nabla \cdot u_{0}=0, \nabla \cdot F_{k 0}=0$. 
Theorem 2.2 Let $(u, F)$ be a solution to (8) in the class (2) for $s=\frac{5}{2}+\gamma$. Assume that $L_{\gamma}(t)$ is defined as above, and that

$$
\int_{0}^{T}\left(L_{\gamma}(\tau)\right)^{-\frac{5}{2}} d \tau<\infty
$$

where $L_{\gamma}(t)=\min \left\{L,\left(\frac{\|(\nabla \times u)(t)\|_{C \gamma}}{\left\|u_{0}\right\|_{L^{2}}}\right)^{-\frac{2}{2 \gamma+5}},\left(\frac{\|(\nabla \times F)(t)\|_{C \gamma}}{\left\|F_{0}\right\|_{L^{2}}}\right)^{-\frac{2}{2 \gamma+5}}\right\}$. Then there exists a finite positive constant $C_{\gamma}=O\left(\gamma^{-1}\right)$ independent of $(u, F)$ and $t$ such that

$$
\|(u, F)\|_{H^{s}} \leq\left\|\left(u_{0}, F_{0}\right)\right\|_{H^{s}} \exp \left\{C_{\gamma}\left\|\left(u_{0}, F_{0}\right)\right\|_{L^{2}} \int_{0}^{t}\left(L_{\gamma}(\tau)\right)^{-\frac{5}{2}} d \tau\right\}
$$

holds for $0 \leq t \leq T$.

This system arises in the Oldroyd model for an ideal viscoelastic flow, i.e. a viscoelastic fluid whose elastic properties dominate its behavior. Here $F=F(x, t) \in \mathbb{R}^{3 \times 3}$ represents the local deformation gradient of the fluid. The blow-up criterion of the ideal viscoelastic system can be found in [11] and references therein.

\section{Proof of Theorem 2.1}

For the proof of our main result, firstly we give some properties about the gradient of velocity. Recall that the full gradient of the velocity, $\nabla u$, can be decomposed into symmetric and antisymmetric parts,

$$
\nabla u=D u^{+}+D u^{-}
$$

where

$$
D u^{ \pm}=\frac{1}{2}\left(\nabla u \pm \nabla u^{T}\right)
$$

$D u^{+}$is called the deformation tensor.

In the following lemmas, we recall some important properties of $D u^{+}$and $D u^{-}$without proof $[7,8]$.

Lemma 3.1 For both the symmetric and the antisymmetric parts $D u^{+}, D u^{-}$of $\nabla u$, the $L^{2}$ bound

$$
\left\|D u^{ \pm}\right\|_{L^{2}} \leq C\|\omega\|_{L^{2}}
$$

holds.

The antisymmetric part $\mathrm{Du}^{-}$satisfies

$$
D u^{-} v=\frac{1}{2} \omega \wedge v
$$

for any vector $v \in \mathbb{R}^{3}$. The vorticity $\omega$ satisfies the identity

$$
\omega(x)=\frac{1}{4} P \cdot V \cdot \int \sigma(\hat{y}) \omega(x+y) \frac{d y}{|y|^{3}},
$$


('P.V.' denotes principal value) where $\sigma(\hat{y})=3 \hat{y} \otimes \hat{y}-1$, with $\hat{y}=\frac{y}{|y|}$. Notably,

$$
\int_{S^{2}} \sigma(\hat{y}) d \mu_{S^{2}}(y)=0
$$

where $d \mu_{S^{2}}$ denotes the standard measure on the sphere $S^{2}$.

The matrix components of the symmetric part have the form

$$
D u_{i j}^{+}=\sum_{k} T_{i j}^{k}\left(\omega_{k}\right)=\sum_{k} \Gamma_{i j}^{k} * \omega_{k},
$$

where $\omega_{l}$ are the vector components of $\omega$, and where the integral kernels $\Gamma_{i j}^{k}$ have the properties

$$
\begin{aligned}
& \Gamma_{i j}^{k}(y)=\sigma_{i j}^{k}(\hat{y})|y|^{-3}, \\
& \left\|\sigma_{i j}^{k}\right\|_{C^{1}\left(S^{2}\right)} \leq C, \\
& \int_{S^{2}} \sigma_{i j}^{k}(\hat{y}) d \mu_{S^{2}}(y)=0 .
\end{aligned}
$$

Thus, in particular, $T_{i j}^{k}$ is a Calderon-Zygmund operator, for every $i, j, k \in\{1,2,3\}$.

We can also give the following useful lemma to provide an upper bound of singular integral operator for the incompressible Euler equations in [7].

Lemma 3.2 For $L>0$ fixed, and $\gamma>0$, let $\iota_{\gamma}(t)$ be defined as above. Moreover, let $\omega_{k}$ $(k=1,2,3)$ denote the components of the vorticity vector $\omega(t)$. Then any singular integral operator

$$
T \omega_{k}(x)=\frac{1}{4 \pi} P \cdot V \cdot \int \sigma_{T}(\hat{y}) \omega_{k}(x+y) \frac{d y}{|y|^{3}},
$$

with

$$
\int_{S^{2}} \sigma_{T}(\hat{y}) d \mu_{S^{2}}(y)=0, \quad\left\|\sigma_{T}\right\|_{C^{1}\left(S^{2}\right)}<C,
$$

satisfies

$$
\left\|T \omega_{k}\right\|_{L^{\infty}} \leq C\left\|u_{0}\right\|_{L^{2}} \iota_{\gamma}^{\frac{5}{2}}(t)
$$

for $k \in\{1,2,3\}$ and the constant $C$ independent of $u$ and $t$.

Now we are ready to give a proof of Theorem 2.1, which is based on combining an energy estimate for ideal MHD equations with the estimate of $\left(\|\nabla u\|_{L^{\infty}}+\|\nabla h\|_{L^{\infty}}\right)$.

For $s>\frac{5}{2}$, we recall the definitions of the homogeneous and inhomogeneous Besov norms for $1 \leq p, q \leq \infty$,

$$
\|f\|_{\dot{B}_{p, q}^{s}}=\left(\sum_{j \in \mathbb{Z}} 2^{j q s}\left\|f_{j}\right\|_{L^{p}}^{q}\right)^{\frac{1}{q}}
$$


and

$$
\|f\|_{B_{p, q}^{s}}=\|f\|_{L^{p}}+\left(\sum_{j \in \mathbb{Z}} 2^{j q s}\left\|f_{j}\right\|_{L^{p}}^{q}\right)^{\frac{1}{q}}
$$

where $f_{j}=P_{j} f$ is the Paley-Littlewood projection of $f$ of scale $j$. We take the $B_{2,2}^{s}$ Besov norm of $u(t)$ and $h(t)$; then

$$
\begin{aligned}
& \frac{d}{d t}\left(\|u(t)\|_{B_{2,2}^{s}}^{2}+\|h(t)\|_{B_{2,2}^{s}}^{2}\right) \\
& \quad \leq C\left(\|\nabla u\|_{\infty}+\|\nabla h\|_{L^{\infty}}\right)\left(\|u(\cdot, t)\|_{B_{2,2}^{s}}^{2}+\|h(\cdot, t)\|_{B_{2,2}^{s}}^{2}\right) .
\end{aligned}
$$

Therefore,

$$
\begin{aligned}
& \frac{d}{d t}\left(\|u(t)\|_{B_{2,2}^{s}}+\|h(t)\|_{B_{2,2}^{s}}\right) \\
& \quad \leq C\left(\|\nabla u\|_{\infty}+\|\nabla h\|_{L^{\infty}}\right)\left(\|u(\cdot, t)\|_{B_{2,2}^{s}}+\|h(\cdot, t)\|_{B_{2,2}^{s}}\right) .
\end{aligned}
$$

However, applying the results of Lemma 3.1 and Lemma 3.2 to $u$ and $h$, and by the definition of $l_{\gamma}(t)$, we obtain

$$
\begin{aligned}
\|\nabla u\|_{L^{\infty}}+\|\nabla h\|_{L^{\infty}} & \leq\left\|D u^{+}\right\|_{L^{\infty}}+\left\|D u^{-}\right\|_{L^{\infty}}+\left\|D h^{+}\right\|_{L^{\infty}}+\left\|D h^{-}\right\|_{L^{\infty}} \\
& \leq C_{\gamma}\left(\left\|u_{0}\right\|_{L^{2}}+\left\|h_{0}\right\|_{L^{2}}\right)\left(l_{\gamma}(t)\right)^{-\frac{5}{2}} .
\end{aligned}
$$

Therefore, we get

$$
\begin{aligned}
& \|u(t)\|_{H^{s}}+\|h(t)\|_{H^{s}} \\
& \quad \simeq\|u(t)\|_{B_{2,2}^{s}}+\|h(t)\|_{B_{2,2}^{s}} \\
& \quad \leq\left(\left\|u_{0}\right\|_{B_{2,2}^{s}}+\left\|h_{0}\right\|_{B_{2,2}^{s}}\right) \exp \left\{C\left(\left\|u_{0}\right\|_{L^{2}}+\left\|h_{0}\right\|_{L^{2}}\right) \int_{0}^{t} l_{\gamma}(s)^{-\frac{5}{2}} d s\right\} \\
& \quad \simeq\left(\left\|u_{0}\right\|_{H^{s}}+\left\|h_{0}\right\|_{H^{s}}\right) \exp \left\{C\left(\left\|u_{0}\right\|_{L^{2}}+\left\|h_{0}\right\|_{L^{2}}\right) \int_{0}^{t} l_{\gamma}(s)^{-\frac{5}{2}} d s\right\}
\end{aligned}
$$

for $s \geq \frac{5}{2}+\gamma$. Thus we complete the proof of Theorem 2.1 .

\section{Competing interests}

The authors declare that they have no competing interests.

\section{Authors' contributions}

The authors declare that the work was realized in collaboration with the same responsibility. All authors read and approved the final manuscript.

\section{Author details}

${ }^{1}$ Department of Mathematics, Shanghai University, Shanghai, 200444, P.R. China. ${ }^{2}$ School of Mathematics and Statistics, Central South University, Changsha, 410083, P.R. China. ${ }^{3}$ Key Laboratory of Metallogenic Prediction of Nonferrous Metals, Ministry of Education, Central South University, Changsha, 410083, P.R. China. 


\section{Acknowledgements}

The first author was supported by the Excellent Young Teachers Program of Shanghai, Doctoral Fund of Ministry of Education of China (No. 20133108120002) and The First-class Discipline of Universities in Shanghai. The research of the third author, who is the corresponding author, was supported by the Natural Science Foundation of China (No. 41204082), the Research Fund for the Doctoral Program of Higher Education of China (No. 20120162120036), Special Foundation of China Postdoctoral Science (No. 2013T60781) and Mathematics and Interdisciplinary Sciences Project of Central South University. Moreover, the authors are grateful to anonymous referees for their constructive comments and suggestions.

Received: 27 February 2014 Accepted: 15 April 2014 Published: 06 May 2014

\section{References}

1. Majda, AJ: Compressible Fluid Flow and Systems of Conservation Laws in Several Space Variables. Applied Mathematical Sciences, vol. 53. Springer, New York (1984)

2. Caflisch, RE, Klapper, I, Steele, G: Remarks on singularities, dimension and energy dissipation for ideal hydrodynamics and MHD. Commun. Math. Phys. 184, 443-455 (1997)

3. Beale, JT, Kato, T, Majda, AJ: Remarks on the breakdown of smooth solutions for the 3-D Euler equations. Commun. Math. Phys. 94, 61-66 (1984)

4. Chen, QL, Miao, CX, Zhang, ZF: On the well-posedness of the ideal MHD equations in the Triebel-Lizorkin spaces. Arch. Ration. Mech. Anal. 195, 561-578 (2010)

5. Du, Y, Liu, Y, Yao, ZA: Remarks on the blow-up criteria for three-dimensional ideal magnetohydrodynamics equations. J. Math. Phys. 50, 023507 (2009)

6. Zhang, ZF, Liu, XF: On the blow-up criterion of smooth solutions to the 3D ideal MHD equations. Acta Math. Appl. Sinica (Engl. Ser.) 20, 695-700 (2004)

7. Chen, T, Pavlovic, N: A lower bound on blowup rates for the 3D incompressible Euler equations and a single exponential Beale-Kato-Majda type estimate. Commun. Math. Phys. 314, 265-280 (2012)

8. Constantin, P: Geometric statistics in turbulence. SIAM Rev. 36, $73-98$ (1994)

9. Constantin, P, Fefferman, C, Majda, AJ: Geometric constraints on potentially singular solutions for the 3-D Euler equations. Commun. Partial Differ. Equ. 21, 559-571 (1996)

10. Deng, J, Hou, TY, Yu, X: Improved geometric conditions for non-blowup of the 3D incompressible Euler equation. Commun. Partial Differ. Equ. 31, 293-306 (2006)

11. Hu, XP, Ryan, H: Blowup criterion for ideal viscoelastic flow. J. Math. Fluid Mech. 15, 431-437 (2013)

10.1186/1687-2770-2014-96

Cite this article as: Liu et al.: A single exponential BKM type estimate for the 3D incompressible ideal MHD

equations. Boundary Value Problems 2014, 2014:96

\section{Submit your manuscript to a SpringerOpen ${ }^{\circ}$ journal and benefit from:}

- Convenient online submission

- Rigorous peer review

- Immediate publication on acceptance

- Open access: articles freely available online

- High visibility within the field

- Retaining the copyright to your article 